\title{
Acknowledgement to Reviewers of Pathogens in 2015
}

\author{
Pathogens Editorial Office \\ Published: 22 January 2016 \\ MDPI AG, Klybeckstrasse 64, CH-4057 Basel, Switzerland; pathogens@mdpi.com
}

The editors of Pathogens would like to express their sincere gratitude to the following reviewers for assessing manuscripts in 2015.

We greatly appreciate the contribution of expert reviewers, which is crucial to the journal's editorial decision-making process. Several steps have been taken in 2015 to thank and acknowledge reviewers. Good, timely reviews are rewarded with a discount off their next MDPI publication. By creating an account on the submission system, reviewers can access details of their past reviews, see the comments of other reviewers, and download a letter of acknowledgement for their records. In addition, MDPI has launched a collaboration with Publons, a website that seeks to publicly acknowledge reviewers on a per journal basis. This is all done, of course, within the constraints of reviewer confidentiality. Feedback from reviewers shows that most see their task as a voluntary and mostly unseen work in service to the scientific community. We are grateful to our reviewers for the contribution they make.

\begin{tabular}{lll} 
Altarac, Silvio & Hube, Bernhard & Radicheva, Mariana \\
Andere, Cecilia & Hultgren, Scott & Ramirez-Garcia, Andoni \\
Anderson, Annaliesa S. & Hunstad, David A. & Rauceo, Jason M. \\
Arduino, Matthew & Hussain, Malik & Réfrégier, Guislaine \\
Bedard, Emilie & Hütter, Gero & Riffard, Serge \\
Bem, Reinout Alexander & Iorio, Ronald M. & Rodríguez, Roberto A. \\
Bleackley, Mark R. & Irimia, Daniel & Roques, Pierre \\
Bowen, Richard A. & Jacobsen, Ilse & Sauer, John-Demian \\
Bozkurt, Mehmet & Jenkinson, H.f. & Scarcella, S. \\
Bøås, Håkon & Jjemba, Patrick K. & Schembri, Mark \\
Brady, Rebecca & Johannsen, Eric & Schildgen, Oliver \\
Brauner, Annelie & Johnson, James R. & Seed, Patrick C. \\
Bridle, Helen & Kai, Hirofumi & Shukla, San Jay \\
Broder, Christopher & Kang, Jaeku & Shuman, Howard \\
Cai, Tommaso & Karim, Shahid & Singer, Steven M. \\
Caimano, Melissa J. & Kassa, Hailu & Smani, Younes \\
Caron, Alexandre & Kirby, Ralph & Snyder, Christopher M. \\
Carpenter, Guy & Krug, Laurie T. & Sobel, Jack \\
Casey, John & Kubes, Paul & Sokurenko, Evgeni V. \\
Cassatella, Marco & Kucharikova, Sona & Sorrell, Tania C. \\
Chevillon, Christine & Latif, Abdalla & Soubies, Sebastien \\
Cloete, Eugene & Leech, John M. & Southgate, Jenny \\
\hline
\end{tabular}


Conti, Heather R.

Cristino, Sandra

Cunnion, Kenji M.

Cwiklinski, Krystyna

Czopowicz, Michał

David, Michael Z.

De Haas, C. J. C.

de Swart, Rik

Delecluse, Henri-Jacques

Di Giovanni, George D.

Dobrindt, Ulrich

Embers, Monica

Ernst, Beat

Espinosa-Urgel, Manuel

Falkinham, Joseph O.

Fang, Rong

Farrell, Paul

Felipe, Ionice

Fidel, Paul L.

Flemming, Hans-Curt

Foley, Steven

Fooks, Anthony R

Foster, Timothy J.

Foxman, Betsy

Furneri, Pio Maria

Gaffen, Sarah

Gargano, Julia W.

Geerlings, Suzanne E.

Girones, Rosina

Godaly, Gabriela

Gómez, Marisa

Hains, David

Hajishengallis, George

Harris, Patrick

Hayashi, Tetsuya
Leibundgut-Landmann, Salome

Leoni, Erica

Lionakis, Michail

Lipke, Peter N.

Liu, Hung J.

Lorenzo-Gómez, M. F.

$\mathrm{Lu}$, Jingrang

Lukashevich, Igor

Lynne, Aaron

Maisch, Tim

Matsumura, Yasufumi

Miethke, Thomas

Minardi, Daniele

Miriagou, V.

Mitchell, Jennifer

Mobley, Harry L. T.

Monaco, Monica

Montgomery, Christopher P.

Mukhopadhyay, Suman

Muthumani, Kar

Nakagawa, Mayumi

Nguyen, Helen

Nickels, Joseph T

Nicolle, Lindsay E.

Nunberg, Jack

Pallen, Mark

Pancholi, Vijay

Papatsiros, Vassilios G.

Park, Hayoung

Peters, Brian M.

Petzke, Mary

Piatek, Rafał

Pitkanen, Tarja

Polyak, Steven

Prévost, Gilles
Spencer, John David

Spilki, Fernando

Stout, Janet

Sullivan, Donna C.

Sullivan, Matthew J.

Suthar, Mehul

Svanborg, Catharina

Svard, Staffan

Swidergall, Marc

Symonds, Erin M.

Szpirer, Cedric

Tang, Jianming

Tempesta, Maria

Teruo, Yamashita

Thiry, Etienne

Tisa, Louis

Tommassino, Massimo

Valero, M. Adela

Van Den Hoogen, Bernadette G.

Wagenaar, Jaap A

Wardenburg, Juliane

Welling, Mick

Witte, Wolfgang

Woodruff, Trent $\mathrm{M}$.

Yamaguchi, Nobuyasu

Yang, Liang

Hayward, S. Diane

Heinekamp, Thorsten

Henderson, Jeffrey P.

Holmes, Mark

Pruden, Amy

Psaroulaki, Anna

Purnell, Sarah

Quindós, Guillermo 